\title{
INVESTIGACIONES
}

\section{La actitud hacia la enseñanza y aprendizaje de la ciencia en alumnos de Enseñanza Básica y Media de la Provincia de Llanquihue, Región de Los Lagos-Chile}

\author{
The attitude of elementary and high school students, towards \\ the teaching and learning of science from the Province \\ of Llanquihue, Los Lagos Region-Chile
}

\begin{abstract}
A atitude frente ao ensino e à aprendizagem da ciência de alunos da educação primaria e media da Província de Llanquihue, Região de Los Lagos-Chile
\end{abstract}

\author{
V. Hernández, E. Gómez, L. Maltes, M. Quintana, F. Muñoz, \\ H. Toledo, V. Riquelme, B. Henríquez, S. Zelada, E. Pérez.
}

Universidad de Los Lagos, Campus Puerto Montt. Chinquihue Km. 6, Puerto Montt. 65-322522 vhernand@ulagos.cl. Verónica Hernández Rosas. Proyecto FONDEF D05I10190

\begin{abstract}
RESUMEN
Esta investigación caracteriza la actitud de los alumnos respecto a la enseñanza y aprendizaje de la ciencia en distintos tipos de establecimientos educacionales. Se entiende la actitud desde cinco dimensiones, todas éstas vinculadas construyen la actitud escolar. Entre los hallazgos encontrados la piedra de tope siguen siendo las didácticas utilizadas en el aula, las cuales desmotivan a los alumnos y los alejan del quehacer científico. Es importante considerar la actitud positiva de los alumnos en cuanto a utilizar el entorno natural como elemento didáctico, creando esto una nueva demanda respecto a la innovación didáctica en el aula y fuera de ella; además de resignificar el entorno, esto permite establecer un vínculo directo con las metodologías constructivistas, teoría de base de la educación en nuestro país.
\end{abstract}

Palabras clave: actitud, enseñanza de la ciencia, didáctica, entorno natural.

\begin{abstract}
The following research characterizes the students' attitude towards the teaching and learning of science in different educational establishments (private, subsidized and public). The attitude is understood from five dimensions and these linked together build up the students' attitude. Among the findings, the teacher's didactics used in the classroom continues being the main problem that discourages and moves the students away from science. It is important to consider the students' positive attitude regarding the use of the natural environment as a didactic resource. This creates a new need regarding the didactic innovation inside and outside the classroom. Moreover, it gives a new significance to the environment, which allows establishing a direct connection to the constructivist methodologies, the fundamental theory of the educational system in our country.
\end{abstract}

Key words: attitude, science teaching, didactics, natural environment.

\section{RESUMO}

Esta investigação caracteriza a atitude dos alunos com respeito ao ensino e à aprendizagem da ciência em diferentes tipos de estabelecimentos educacionais. Entende-se a atitude desde cinco dimensões, todas estas vinculadas, constroem a atitude escolar. Entre os achados encontrados, a pedra de topo segue sendo as didáticas utilizadas na sala de aula, as quais desmotivam aos alunos e os afastam das tarefas científicas. É importante considerar a atitude positiva dos alunos em quanto a utilizar o meio natural como elemento didático, criando isto uma nova demanda com respeito à inovação didática dentro e fora da sala de aula. Além de dar um significado novo ao meio, isto permite estabelecer um vínculo direto com as metodologias construtivistas, que são a teoria de base da educação em nosso país.

Palavras-chave: atitude, ensino da ciência, didática, meio natural. 


\section{INTRODUCCION}

La educación en Chile ha superado grandes dificultades a través de los años. Desde la restauración de la democracia se ha visto un cambio progresivo en cuanto al número de estudiantes insertos en el sistema educativo (Cox, 1997); no obstante, la calidad en cuanto a los aprendizajes de los alumnos es un tema que está en el centro de la discusión. “...la gran mayoría de nuestros niños y jóvenes tiene acceso a la educación básica y media, pero no todos tienen el mismo derecho a contar con una educación de calidad. La calidad parece estar reservada sólo para quien pueda pagarla..." (Colegio de Profesores, 2006: 7).

Uno de los problemas más relevantes de la enseñanza es lo descontextualizado que se presenta el conocimiento, toda vez que las distintas disciplinas se realizan como subsectores aislados y no se contextualizan a los requerimientos e intereses de los estudiantes, además no se ajustan a los nuevos paradigmas constructivistas, todo lo cual impide el logro de aprendizaje, motivación e interés por parte del alumnado.

Sin lugar a dudas, la contextualización de los aprendizajes se torna más importante en aquellas disciplinas que deben tener una aplicación concreta, debido a la complejidad práctica de los conocimientos que las componen. Este es el caso de la enseñanza de la ciencia, la cual debe facilitar el aprendizaje de los alumnos, los que la ven “...como algo aburrido, principalmente porque en las clases tienen problemas de comprensión; ello trae como resultado que los alumnos tengan bajo rendimiento, poco a poco se desmotiven, se alejen de la ciencia y pierdan el interés”. (Rioseco, Romero, 1997: 1).

$\mathrm{Si}$ bien es cierto los docentes se esmeran por entregar una educación de calidad, al fin y al cabo no logran alcanzar este gran eslabón, puesto que enseñar ciencia en los establecimientos educacionales de Chile es prácticamente un desafío para todos los docentes del área, debido a que las metodologías usadas cotidianamente no logran motivar a los estudiantes ni se ajustan a los nuevos métodos de enseñanza propuestos por la teoría constructivista. Como afirma M. Cereijido (1997), “en América Latina no tenemos ciencia (aunque en algunos casos exista muy buena investigación científica), el problema es mucho más profundo que una cuestión de experimentos y demostraciones. No es creer o reventar: es demostrar, preguntar, inquietar". (Golombek, 2008: 13).

Desde el punto de vista constructivista, "la finalidad última de la intervención pedagógica es desarrollar en el alumno la capacidad de realizar aprendizajes significativos por sí solo, en una amplia gama de situaciones y circunstancia (aprender a aprender)" (Coll, 1988: 133). Desde ahí, la enseñanza de la ciencia se debe contextualizar acorde a las realidades de los alumnos, a sus entornos inmediatos, en los que ellos puedan intervenir creando y solucionando problemas de la vida cotidiana.

Es relevante considerar que "...la aplicación de estrategias metacognitivas inadecuadas puede ayudar a entender algunas de las conductas típicas de los alumnos en tareas de aprendizaje de las ciencias" [...] "Los alumnos parecen quedarse satisfechos con el poder explicativo de sus ideas previas a pesar de que la información que reciben de sus profesores y libros de texto con frecuencia contradice estas ideas previas" (Campanario, Cuerva, Moya y Otero, 1998: 41).

Según lo establecen Pozo y Gómez (2004: 17), “Cunde entre los profesores de ciencia una creciente sensación de desasosiego, de frustración, al comprobar el limitado éxito de sus esfuerzos docentes. En apariencia los alumnos cada vez aprenden menos y 
se interesan menos por aprender...” Después de aplicados muchos procesos de reforma se puede decir que aún se comparten visiones empobrecidas y deformadas respecto a la enseñanza escolar de la actividad científica (Gil et al., 1991; Porlán, 1993), lo cual no favorece en los estudiantes una actitud positiva al estudio de la ciencia.

Es por tanto necesario hacer innovaciones de índole educativa, que se realicen directamente en las aulas, que permitan enseñar ciencia bajo contenidos más pertinentes a los intereses y espacios de los alumnos, donde ellos puedan dar explicaciones científicas a experiencias de la vida diaria, donde a través de este proceso puedan mejorar su calidad de vida, siendo capaces de transformar sus entornos y entiendan las consecuencias de esto.

\section{LA ACTITUD: IDEAS INICIALES}

El concepto actitud proviene de la palabra latina "actitudo", definiéndose desde la psicología como aquella motivación social de las personas que predisponen su accionar hacia determinadas metas u objetivos. Existen actitudes personales que guardan relación con características particulares de los individuos, mientras que existen ciertas actitudes sociales que inciden en las conductas de un grupo o colectivo. Además se establece que para desarrollar una actitud adecuada al proceso de aprendizaje es necesario intervenir: (i) Aspectos cognitivos (conocimientos y creencias), (ii) Aspectos afectivos (sentimientos y preferencias) y (iii) Aspectos conductuales (intenciones o acciones manifiestas) (Rodríguez, 1991). Todo esto, además debe estar vinculado con las múltiples experiencias y relaciones que las personas o grupos hayan ido acumulando a lo largo de su historia de vida.

También es importante señalar que las representaciones sociales son fundamentales respecto a la actitud de los sujetos (Moscovici, 1986), especialmente las que se instalan como mitos en nuestra sociedad; por ejemplo, la religión; aquí se puede mencionar la representación social (escolar) respecto al aprendizaje de la ciencia y las matemáticas.

Según la psicología social, la actitud es como una organización relativamente duradera de creencias (inclinaciones, sentimientos, prejuicios o tendencias, nociones preconcebidas, ideas, temores y convicciones) aprendidas acerca de un objeto, situación o experiencias dadas, las cuales predisponen a reaccionar de una manera determinada. Es decir, que podemos considerar a la actitud como la predisposición de una persona a reaccionar favorable o desfavorablemente hacia un objeto, que puede ser una cosa, otra persona, una institución, lo cual evidentemente puede provenir de la representación social que se ha construido acerca de ese objeto (Moscovici, 1986; Escudero, 1985).

De igual modo, según Allport (1968) la actitud se establece como el vínculo existente entre el conocimiento adquirido de un individuo sobre un objeto y la acción que realizará en el presente y en el futuro en todas las situaciones en que corresponde; la actitud tiene directa relación con la visión que tengamos del mundo que nos rodea, en tanto se modifica sólo cuando cambiamos nuestras creencias y percepciones respecto del mundo o las áreas específicas de análisis. Como las creencias se construyen en espacios sociales, también podemos considerar la existencia de actitudes asociadas a ciertos grupos, como por ejemplo, en el sistema educativo. 


\section{INNOVACION EN DIDACTICA DE LA CIENCIA}

Esta investigación postula que el gran problema de la enseñanza y aprendizaje de la ciencia guarda relación con la insuficiente experimentación, así como el escaso nivel de pertinencia de la didáctica con la realidad de los educandos, por consiguiente la poca utilidad práctica que se le da al conocimiento adquirido, lo que genera bajos niveles de aprendizajes, afectando directamente la motivación del estudiante hacia el área científica, y a su vez el número deficiente en la formación de científicos y el poco desarrollo de dicha área en la región.

También este escaso nivel de pertinencia de la enseñanza de la ciencia está dado porque frecuentemente los docentes no profundizan en el análisis de las situaciones de enseñanza planteadas, quedándose en la mera intención de abordar contenidos científicos, lo que no contribuye a lograr la transferencia adecuada de los contenidos escolares al análisis e interpretación de los fenómenos y situaciones de la vida real. "Si bien los temas propuestos por el currículo pueden ser relevantes para desarrollar habilidades para la vida, las formas de enseñar establecen la diferencia, a la hora de evaluar los resultados de los aprendizajes" (Leymonié Sáenz, 2009: 66).

Entonces la utilización de los entornos inmediatos como elementos didácticos motiva a los alumnos y acrecienta una actitud positiva. Un ejemplo que se encuentra en desarrollo por el presente equipo de investigación es la utilización del entorno acuático como elemento didáctico; de resultar exitosa la experiencia, nos permitirá también pensar que posteriormente podemos enseñar ciencias en base a los entornos mineros, del agro, entre otros.

El equipo de investigación del proyecto reconoce una serie de variables que intervienen en la actitud de los alumnos y por tanto las bases de la investigación en esta área son:

1. Autoconcepto académico (Aut): Papalia 2001 entiende el autoconcepto como el sentido de sí mismo, es decir, la imagen mental descriptiva y evaluativa de las capacidades y rasgos de sí. Éste se forma a partir de las experiencias y de los comentarios y apoyo que recibe de las personas de su entorno. Desde esta premisa, el autoconcepto académico influye en el rendimiento y viceversa; puesto que un alumno que confía en sus aptitudes se esforzará por conseguir buenos resultados y dichos buenos resultados contribuirán a fortalecer la imagen de buen estudiante, por ello, lo más probable es que mantenga esta visión, ya que se sentirá confiado de sí mismo. En este sentido, la percepción que los individuos tienen de su valía como estudiantes se forma a partir de sus experiencias personales y con su entorno, influenciado por los resultados escolares obtenidos y/o su imagen como aprendiz. Se superpone, además, con la autopercepción de aptitud y la habilidad para aprender (Tapia, 1997). Otro concepto importante es la motivación académica, el cual es elaborado a través de las experiencias de aprendizaje y se basa en esquemas mentales que asocian el objeto con experiencias positivas (Bandura, 1986; Köller, 2001; Marsh, 2005).

2. Actitud hacia el aprendizaje de la ciencia (AppCs): Son diversas las investigaciones que señalan la existencia de una relación entre las creencias y la actuación de los profesores de ciencias (Porlán, 1999; Gess-Newsome, 1999; Haney y Mcarthur, 2002; Bryan, 2003; Moreno y Azcárate, 2003; Wu y Krajcik, 2006; Trumbull, Scarano y Boney, 2006). Entre los profesores de ciencia se nota una creciente sensación 
de desasosiego, de frustración, debido al limitado éxito de sus esfuerzos docentes. Asimismo, los alumnos cada vez aprenden menos y se interesan menos por aprender (Pozo y Gómez, 2004; Rioseco y Romero, 2000). Después de aplicados muchos procesos de reforma se puede ver que las visiones empobrecidas y deformadas que proporciona frecuentemente la enseñanza escolar de la actividad científica no favorecen en los estudiantes una actitud favorable al estudio de la ciencia (Gil, 1991; Porlán, 1993); las actitudes negativas suelen incrementarse con los años de escolaridad (Gil, 1994).

3. Actitud hacia el uso de distintos ecosistemas como medio para el aprendizaje de la ciencia (UtEc): Hasta este momento la enseñanza de la ciencia ha sido en nuestros sistemas exclusivamente libresca, verbalista, desinteresada, descontextualizada de la vida social y centrada en la memorización de datos puntuales, cuyo significado formativo y relevancia social es para decir lo menos, precario y altamente cuestionable (Silva Castro, 2003). Reconociendo que la enseñanza de la ciencia no logra despertar interés en los alumnos (Pozo y Gómez, 2004; Rioseco y Romero, 2000) es importante tener en cuenta que el aprendizaje utilizando la naturaleza como sala de clases o laboratorio permite a los alumnos desenvolverse en actividades de mayor complejidad, empleando el entorno, indagando y experimentando, lo que presenta posibilidades reales de aplicación de didácticas (Payne 1985). Para muchos alumnos, saber que van a trabajar fuera del laboratorio ya es un aliciente. A diferencia del trabajo "de verdad", el trabajo de campo es divertido, es "biología en acción", sin la simplicidad aséptica y "conformidad con las expectativas" del laboratorio. Además, si se realiza en el entorno local, puede crear en ellos, como futuros ciudadanos, vínculos fuertes con la vida adulta, al llegar a interesarlos por su región y a comprometerse con su desarrollo (Roger, 1998: 4).

4. Actitud hacia la institucionalidad escolar (clases de ciencias) (Inst): Según lo establece Martín (2002) la finalidad de la enseñanza de las ciencias ha ido variando a lo largo de las últimas décadas, mientras las primeras reformas perseguían la creación de "pequeños científicos" gracias a los nuevos métodos didácticos que ponían el énfasis en "la Ciencia como interrogación" o "el aprender haciendo" (Matthews, 1991) donde el enfoque didáctico estaba basado en la metodología científica y desarrollaron taxonomías de objetivos científicos que aspiraban a conseguir determinadas competencias en cuanto a procedimientos y actitudes (Porlán, 1993). Posteriormente, la Didáctica de las Ciencias recibió nuevas influencias provenientes del campo de la epistemología y de la psicología del aprendizaje, una marcada tendencia a investigar sobre las concepciones que los alumnos tienen acerca de los fenómenos naturales antes de recibir una enseñanza científica formal. Preconceptos, ideas previas, marcos conceptuales alternativos y concepciones espontáneas son algunas de las denominaciones que fueron surgiendo (Pozo y Gómez Crespo, 2001). Actualmente se desarrollan con fuerte impulso el enfoque Ciencia-Tecnología-Sociedad y el enfoque de la Educación Ambiental (Leymonié Sáenz, 2009). Todo el desarrollo de la enseñanza de las ciencias pareciera no estar plasmado aún en la didáctica que se realiza en el aula, toda vez que lo confirma el nivel de aprendizaje obtenido por los alumnos.

5. Aprendizaje escolar (App): Un componente importante en el aprendizaje es la actitud que asumen los alumnos ante el proceso, esto trata de la predisposición por aprender, permitiendo abordar de forma crítica su propio proceso de aprendizaje; también 
pasa a ser importante que ellos valoren el aprendizaje como parte de su desarrollo personal. Desde lo antes mencionado, el aprendizaje es la relación existente entre la actitud hacia la institucionalidad escolar y el aprendizaje de la ciencia. Esta actitud es formada por toda la comunidad escolar (profesores, pares, padres, ambiente de aprendizaje y autoconcepto) y está íntimamente ligada con el logro de los alumnos, la motivación y el interés. Según Morrel \& Lederman (1998) esta actitud hacia la escuela y la ciencia va decreciendo con los grados superiores (Glick, 1970; Haladyna, et al., 1983; Darom \& Rich, 1988).

\section{DISEÑO METODOLOGICO}

La investigación es de tipo cualitativa; el fenómeno de estudio es la actitud respecto a la enseñanza y aprendizaje de la ciencia en alumnos de NB5 $\left(7^{\circ}\right)$ de Educación General Básica y NM1 $\left(1^{\circ}\right)$ de Educación Media.

La muestra está constituida por 414 alumnos, de estos 156 corresponden a $7^{\circ}$ Básico y 258 a $1^{\circ}$ medio; esta muestra representa a todos los tipos de establecimientos educacionales según la siguiente distribución: EE4 (Particular), EE2 (Particular Subvencionado), EE1, EE3, EE5, EE6 (Municipales), además representan a dos comunas: Puerto Montt y Calbuco, de la provincia de Llanquihue, Región de Los Lagos.

El instrumento utilizado es una Escala Likert ${ }^{1}$, en la cual se desarrollan un conjunto de ítems presentados en forma de afirmaciones o juicios ante los cuales se pide la reacción de los sujetos, por tanto en el análisis se llamarán reactivos; los sujetos deben elegir uno de los cinco puntos de la escala, los cuales tienen un valor preestablecido, posteriormente cada sujeto obtiene una puntuación respecto a sus propias opiniones y estos se suman con las demás puntuaciones obtenidas por los otros sujetos. La Likert está compuesta por cinco dimensiones y cada una está compuesta por seis reactivos, algunos positivos o negativos, los cuales fueron seleccionados a través del método de comparación entre el cuartil de mayor puntaje y el cuartil de menor puntaje para cada dimensión, seleccionando aquellos reactivos que mostraban comportamientos más disímiles en la comparación; la validación del instrumento se realizó en distintos establecimientos educacionales.

Las dimensiones son las siguientes:

1. Autoconcepto académico (Aut)

2. Actitud hacia el aprendizaje de la ciencia (AppCs)

3. Actitud hacia el uso de distintos ecosistemas como medio para el aprendizaje de la ciencia (UtEc)

4. Actitud hacia la institucionalidad escolar (clases de ciencias) (Inst)

5. Actitud hacia el aprendizaje escolar (App)

1 Escalamiento desarrollado por Rensis Likert a principios de los años 30 (Hernández, 1998: 262), es una escala de actitud. Summers (1982) define el término actitud como la “...suma total de inclinaciones y sentimientos, prejuicios o distorsiones, nociones preconcebidas, ideas, temores, amenazas y convicciones de un individuo acerca de cualquier asunto específico" (p. 158). La actitud se expresa por medio de opiniones, por ejemplo, una persona que expresa su opinión sobre la caída del muro de Berlín y la reciente desintegración de la Unión de Repúblicas Socialistas Soviéticas refleja una actitud específica sobre los hechos referidos. 
Los rangos que utiliza la escala ordinal son:

1: Muy en Desacuerdo

2: En Desacuerdo

3: Indeciso

4: De acuerdo

5: Muy de Acuerdo

El análisis de resultados se realizará por cada dimensión del instrumento; los rangos seleccionados para el análisis serán de acuerdo y muy en desacuerdo, los cuales permiten evidenciar la actitud positiva.

\section{RESULTADOS}

La edad de los alumnos se encuentra en una media de 13,5 años, en tanto los alumnos de $7^{\circ}$ básico tienen un promedio de edad 12,3 años y los de $1^{\circ}$ medio un 14,4 .

\section{ANALISIS POR DIMENSION}

1. AUTOCONCEPTO ACADEMICO (Aut):

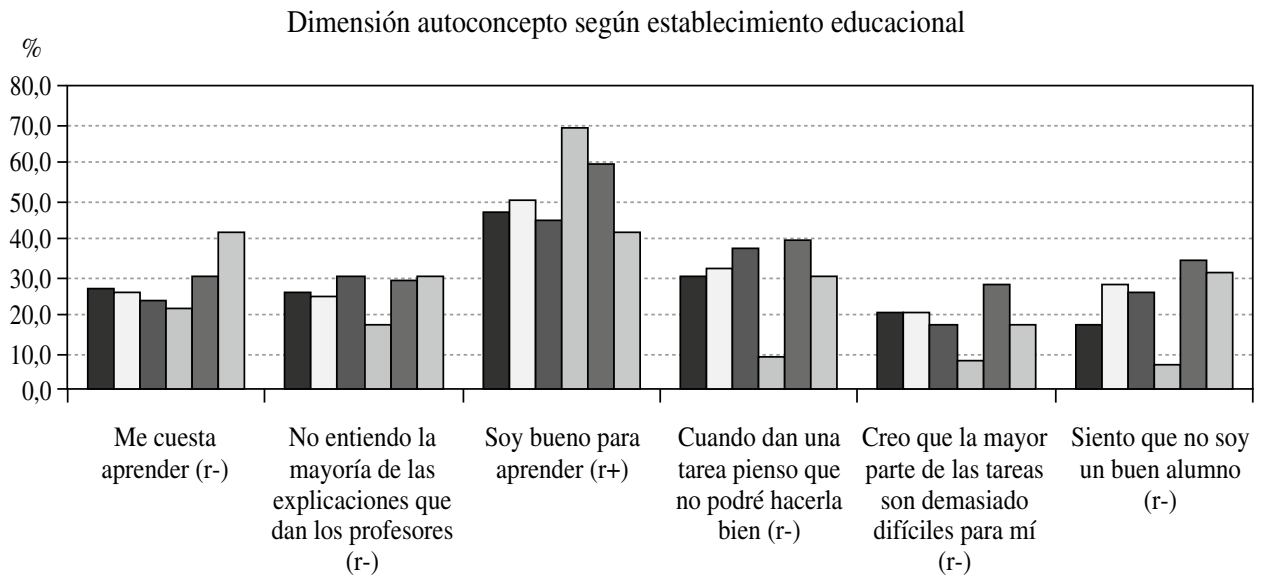

\begin{tabular}{|c|c|c|c|c|}
\hline EE 1 & $\square \mathrm{EE} 2$ & 口EE 3 & $\square \mathrm{EE} 4$ & पEE 5 \\
\hline
\end{tabular}

En cuanto al autoconcepto académico, se puede determinar que la actitud de los alumnos es medianamente positiva, si consideramos que los reactivos negativos tienden a presentar un bajo porcentaje respecto a los positivos, ej. "Soy bueno para aprender" $(\mathrm{r}+)$ todos los establecimientos superan $41,0 \%$, en tanto en los reactivos negativos no superan $41,1 \%$ "me cuesta aprender", sí, es necesario destacar la disparidad que existe entre los establecimientos educacionales especialmente según nivel socioeconómico ejemplo EE 6: r-1 41,4\%, EE 5: r-6 34,0\%, en tanto en el colegio particular pagado EE 4 los reactivos negativos tienen una actitud positiva $(\mathrm{r}-4 \mathrm{8,0 \%})$. 
2. ACTITUD HACIA EL APRENDIZAJE DE LA CIENCIA (AppCs):

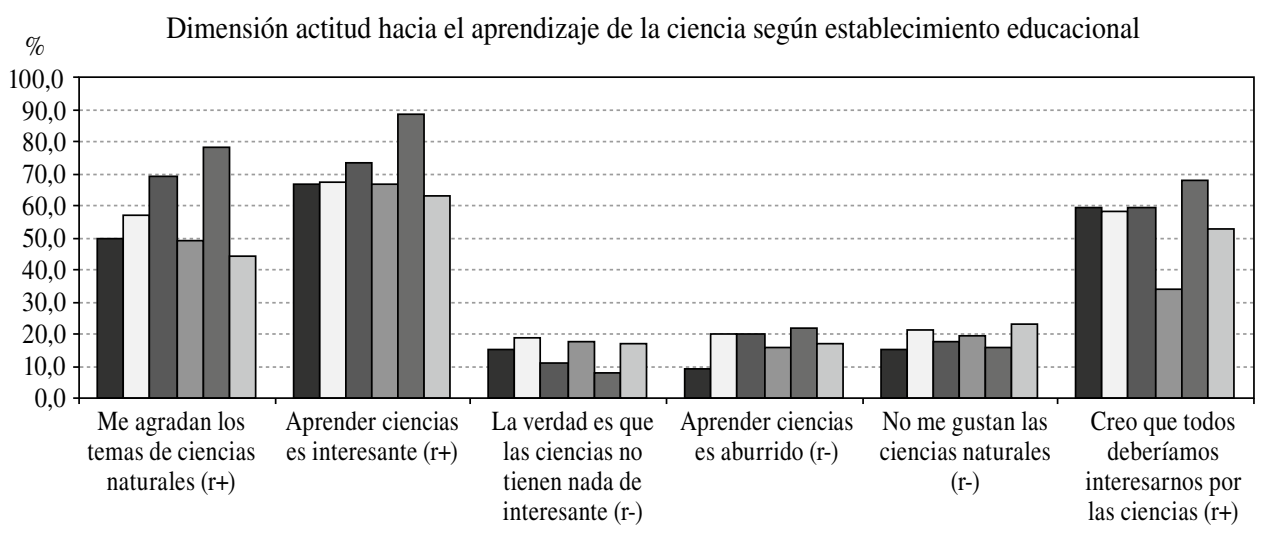

\begin{tabular}{|llllll|}
\hline$\square \mathrm{EE} 1$ & $\square \mathrm{EE} 2$ & $\mathrm{EE} 3$ & $\mathrm{EE} 4$ & $\mathrm{EE} 5$ & $\square \mathrm{EE} 6$
\end{tabular}

La actitud de los alumnos tiene una clara tendencia a ser positiva, especialmente en cuanto a la alta puntuación de los reactivos positivos, como "aprender ciencia es interesante" $(\mathrm{r}+2)$, donde el EE 5 alcanza una actitud positiva con un porcentaje superior al 85\% y el EE 3: 70\%, en tanto el EE 4 presenta una actitud con una tendencia más negativa, lo cual se manifiesta en el $\mathrm{r}+6$ con un $34 \%$.

\section{ACTITUD HACIA EL USO DE DISTINTOS ECOSISTEMAS COMO MEDIO PARA EL APRENDIZAJE DE LA CIENCIA (UtEc):}

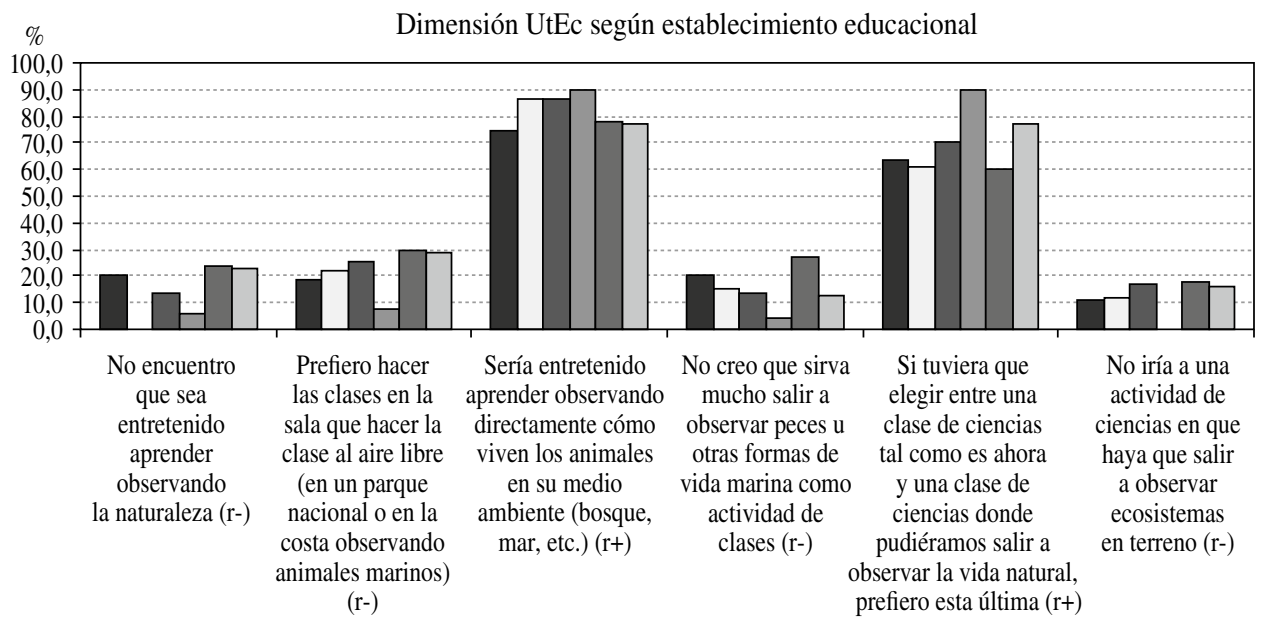

$\square$ EE $1 \square$ EE $2 \quad \square$ EE $3 \quad \square$ EE $4 \quad \square$ EE $5 \quad \square$ EE 6


Respecto a la actitud de los alumnos hacia un cambio de metodología que considere la aplicación del medio natural como elemento didáctico ésta es positiva, especialmente salidas a terreno a parques nacionales, aprender de los animales, del medio ambiente, entre otras, esto implica una actitud bastante negativa respecto a como se realizan las actividades de aula hoy, destaca en el $\mathrm{r}+5$ donde el EE 4 alcanza un 90,2\%, de igual forma en el $\mathrm{r}+3$ con un 90,2\%. En tanto en los reactivos negativos, los establecimientos con la actitud más positiva hacia las clases tradicionales son el EE 5: (r- 4 27,5\%) y el EE 6: (r- 2 28,6\%).

\section{ACTITUD HACIA LA INSTITUCIONALIDAD ESCOLAR (CLASES DE CIENCIAS) (Inst):}

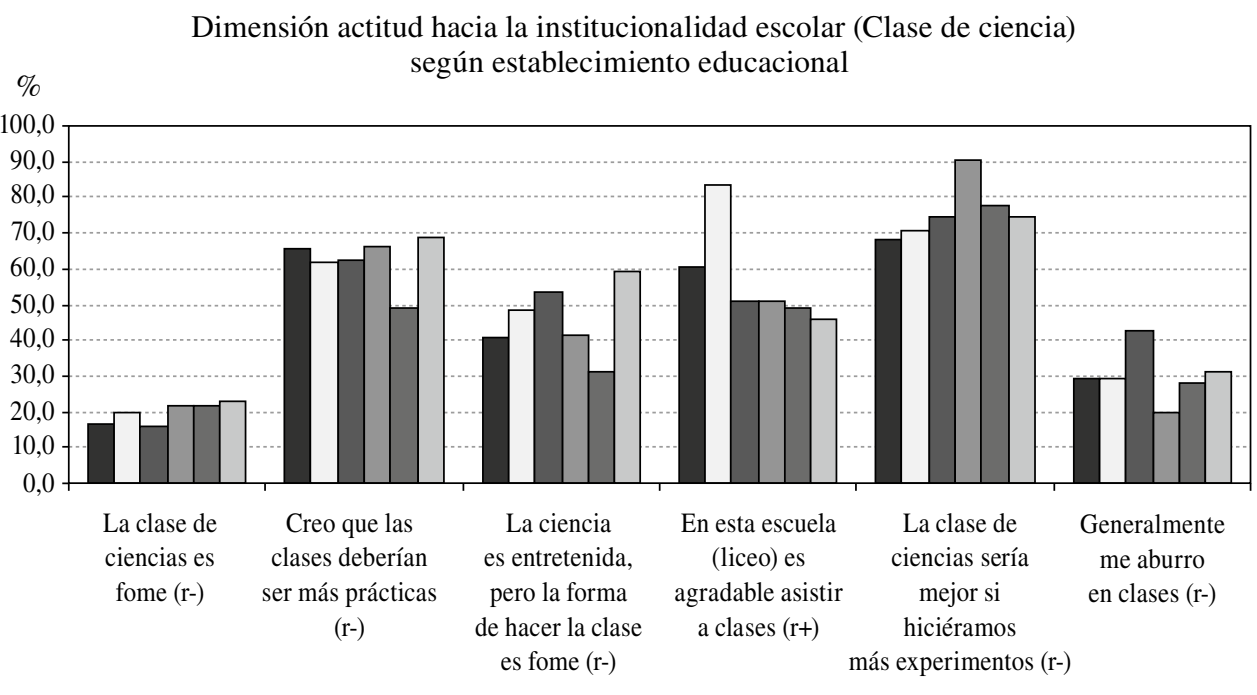

$$
\begin{array}{|llllll}
\hline \mathrm{EE} 1 & \square \mathrm{EE} 2 & \square \mathrm{EE} 3 & \square \mathrm{EE} 4 & \square \mathrm{EE} 5 & \square \mathrm{EE} 6
\end{array}
$$

En la presente dimensión existe una actitud muy negativa por parte de los alumnos hacia las clases de ciencias que hoy se desarrollan en los establecimientos educacionales; se reconoce que "la ciencia es entretenida pero la forma de hacerla es fome" r- 3 EE 6: 59,4\%, EE 3: 53,3\%, los alumnos a su vez presentan una actitud positiva a la realización de experimentos r-5 EE 4: 90,2\%, a su vez las clases deberían ser más prácticas (r- 2) la EE 1: 65,8\%, en cuanto a la actitud de los alumnos respecto a lo agradable que es asistir a clases $(\mathrm{r}+4)$ en el EE 2 presenta la actitud más favorable con un $83,3 \%$. 
5. ACTITUD HACIA EL APRENDIZAJE ESCOLAR (App):

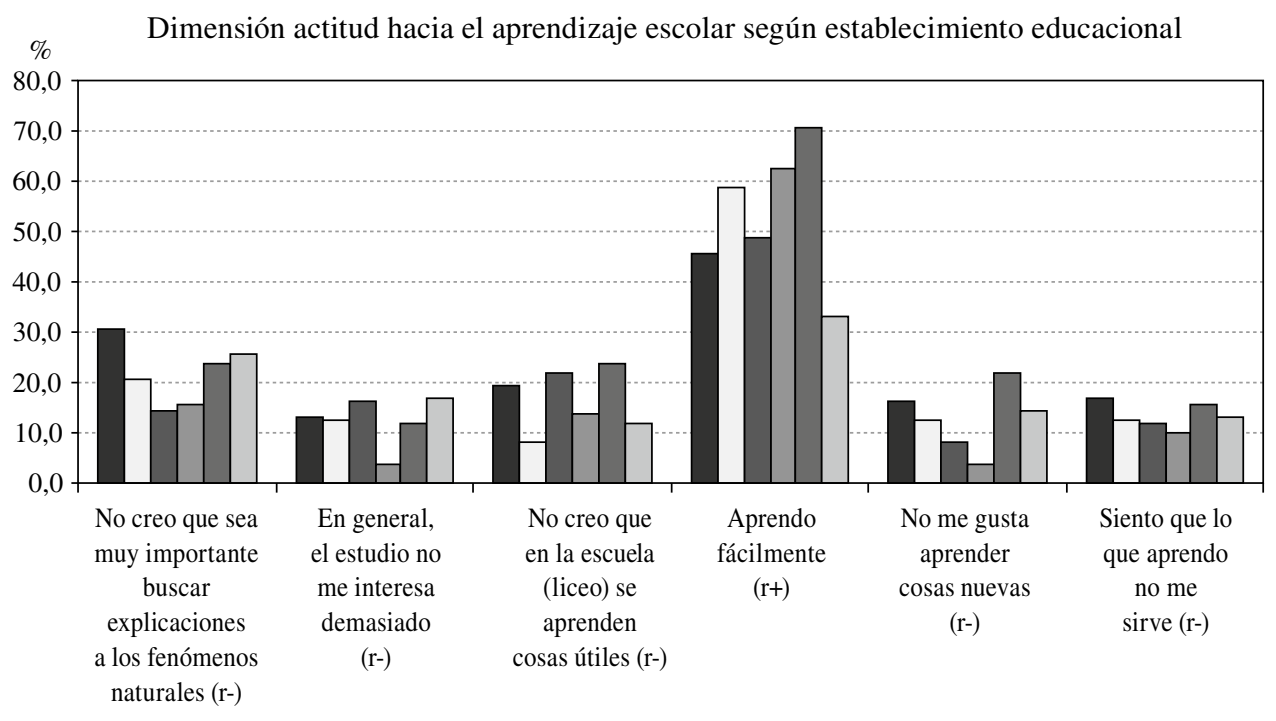

$\square$ Melipulli $\square$ Bosque Nativo $\square$ Politécnico Calbuco $\square$ American School $\square$ Eulogio Goycolea $\square$ Mirasol

La actitud de los alumnos hacia el aprendizaje escolar es positiva, toda vez que los r- tienen una porcentaje muy bajo r- 2, EE 4: 3,9\%, EE 1: 12,8\%, EE 2: 12,8\%, en tanto el r+ 4 EE 5: 70,6\%, siendo la actitud más negativa el EE 6: 32,9\%.

\section{CONCLUSIONES}

Los resultados encontrados en la presente investigación no están muy acordes a nuestras percepciones preliminares ni a los hallazgos investigativos anteriormente descritos, toda vez que suponíamos que los alumnos tenían una actitud muy negativa hacia el aprendizaje de la ciencia.

$\mathrm{Si}$ analizamos por dimensión podemos determinar que en cuanto al autoconcepto académico la actitud negativa es más recurrente en los establecimientos educacionales que acogen a los alumnos de los niveles socioeconómicos más bajos, relacionando este antecedente más con problemas de orden social que problemas relacionados al sistema educativo; sí es importante destacar que una actitud negativa respecto a su propia imagen y capacidad de aprender influirá negativamente respecto del interés de los alumnos por el sector específico de la ciencia y por todos los sectores del currículum escolar.

Respecto a la dimensión AppCs, los resultados contradicen lo que la literatura indica. Los alumnos consideran que aprender ciencia es interesante, si vinculamos este resultado a la dimensión UtEc podemos percibir que para los alumnos se torna mucho más interesante aprender ciencias si se utiliza el ecosistema como elemento didáctico, 
traduciendo esto a observaciones del medio ambiente, salidas a terreno, clases al aire libre, entre otros. Desde lo anterior, resulta obvio que la dimensión Inst, clase de ciencia, tenga la actitud más negativa por parte de los alumnos, reconociendo que la ciencia es entretenida, pero la forma de hacerla es muy "fome", lo cual nos permite parafrasear a Castro 2003: la ciencia que se encuentra hoy día en nuestras aulas es exclusivamente libresca, verbalista, desinteresada, descontextualizada de la vida social y centrada en la memorización de datos puntuales, cuyo significado formativo y relevancia social es, para decir lo menos, precario y altamente cuestionable.

$\mathrm{Si}$, con todos los antecedentes anteriores analizamos la última dimensión, la dimensión App es donde los alumnos presentan una actitud muy positiva respecto al aprendizaje escolar.

Entonces ante el análisis de resultados podemos concluir que el cambio de la didáctica, el cambio de las prácticas pedagógicas es un tema de alta prioridad, por tanto iniciativas como la utilización de los entornos naturales (acuáticos, terrestres, forestales, entre otros) a través de reales procesos de enseñanza y aprendizaje, de perfeccionamiento a los profesores en la adquisición de estos nuevos conocimientos, permitirá cambiar la actitud de los alumnos, como los indicadores que hoy día desvalorizan la enseñanza de la ciencia en los establecimientos educacionales, especialmente si consideramos la diversidad de medios naturales que presenta nuestra región, como nuestro país.

\section{REFERENCIAS BIBLIOGRAFICAS}

Alonso Tapia, J. (1997). "Motivar para el aprendizaje. Teorías y estrategias". EDEBE, Barcelona. Allport, G.W. (1968). "The historical background of modern social psychology". En Lindzey, G. \& Aronson, E. (Eds.): Handbook of Social Psychology. Vol. I. Boston: Addison-Wesley.

Bandura, S. (1986). "Social Foundation of Thought and Action: A social cognitive theory". Englewood Cliff, NJ: Prentice-Hall.

Bryan, L. (2003). "Nestedness of beliefs: examining a prospective elementary teachers' belief system about science teaching and learning". Journal of Research in Science Teaching.

Campanario, L., Cuerva M., Moya L. y Otero G. (1998). "La metacognición y el aprendizaje de las ciencias". Investigación e innovación en la enseñanza de las ciencias. Vol. 1. ISBN 8495095-03-3.

Cervini, R. y Gosende, E. (2008). “Características no cognitivas, habitus y progreso del aprendizaje de Matemática en la Educación General Básica (EGB) de la Provincia de Buenos Aires (República Argentina)". Centro Interamericano de Investigaciones Psicológicas y Ciencias Afines. Argentina. Disponible en: http://redalyc.uaemex.mx/redalyc/src/inicio/ArtPdfRed.jsp?iCve=18025201

Cereijido, M. (1996). En América Latina ya podemos investigar, el próximo paso es tratar de hacer ciencia. Interciencia 21(2): 64-70. URL: http://www.interciencia.org.ve

Cereijido, M. (1997). "Por qué no tenemos ciencia". Siglo XXI Editores. México.

Colegio de Profesores de Chile (2006). "La crisis del Sistema Educativo Chileno".

Colegio de Profesores. En OCDE (2004). "Revisión de Políticas Nacionales de Educación". Chile, febrero, Paris. www.opech.cl/movisociales/propuestas/doc_colegio_profes.pdf

Coll, C. (1996). "Aprendizaje escolar y construcción del conocimiento". Paidós, Buenos Aires.

Coll, C. (1988). "Significado y sentido en el aprendizaje escolar. Reflexiones en torno al concepto de aprendizaje significativo". En Infancia y Aprendizaje, No 41, pp. 131-142.

Cox, C. (1997). "La Reforma de la Educación Chilena: Contexto, Contenidos, Implementación". Preal. Santiago de Chile. 
Davidoff, L.L. (1989). "Introducción a la Psicología". 3ª edición. Editorial McGraw-Hill. México.

Darom, E. \& Rich, Y. (1988). Sex differences in attitudes toward school: Student self-reports and teacher perceptions. British Journal of Educational Psychology.

Díaz Barriga, F. y Hernández Rojas, G. (1998). "Estrategias Docentes para un Aprendizaje Significativo: Una interpretación Constructiva. $1^{\text {a }}$ edición. McGraw-Hill/Interamericana Editores. México D.F., México.

Escudero, E. (1985). Las actitudes en la enseñanza de las ciencias: un panorama complejo. En: Revista de Educación. N 278 (septiembre-diciembre).

Gess-Newsome, J. (1999). "Pedagogical content knowledge: An introduction and orientation". En Gess-Newsome, J. \& Lederman, N. (Eds.): Examining Pedagogical Content Knowledge. Dordrecht, Kluwer Academic Publishers.

Gil, D. (1994). "Relaciones entre conocimiento escolar y conocimiento científico. Investigación en la Escuela".

Gil Pérez, D., Carrascosa, J., Furio, C. y Martínez, J. (1991). "La enseñanza de la ciencia en la educación secundaria". Editorial Horsori. Barcelona.

Glick, O. (1970). Sixth graders' attitudes toward school and interpersonal conditions in the classroom. The Journal of Experimental Education.

Golombek, D. (2008). Aprender y Enseñar Ciencias: del laboratorio al aula y viceversa. Fundación Santillana. Primera Edición. Buenos Aires, Argentina.

Haladyna, T., Olsen, R. \& Shaughnessy, J. (1983). Correlates of class attitude toward science. Journal of Research in Science Teaching, 20(4), 311-324.

Haney, J. \& Mcarthur, J. (2002). "Four case studies of prospective teachers' beliefs concerning constructivist practice". Science Education.

Hoffman, L., Paris, S. y Hall, E. "Psicología del Desarrollo Hoy". 6ª edición. Volumen I. Editorial McGraw-Hill. Madrid, España.

Jiménez Aleixandre, M.P. (1992). "Introducción y análisis de modelos. Didáctica de las Ciencias de la Naturaleza". Madrid: MEC.

Joyce, B. y Weil, M. (1985). "Modelos de enseñanza". Madrid: Anaya.

Köller, O. et al. (2001). "Does interest matter? The relationship between academy interest and achievement in mathematics". Journal for Research in Mathematical Education.

Köller, O., Baumert, J. \& Schnabel, K. (2001). "Does interest matter? The relationship between academic interest and achievement in mathematics". Journal for Research in Mathematics Education.

Lev S., Vygotski (2003). El desarrollo de los procesos psicológicos superiores. Segunda edición. Editorial Crítica. Barcelona, España.

Leymonié S.J. (2009). Aportes para la enseñanza de las Ciencias Naturales. UNESCO. Impreso por Salesianos Impresores S.A. Santiago, Chile.

Marsh, H., Trautwein, U., Lüdtke, O., Köller, O. \& Baumert, J. (2005). Academic self-concept, interest, grades, and standardized test scores: reciprocal effects models of causal ordering. Child Development.

Martín Díaz, M. (2002). “Enseñanza de las ciencias ¿Para qué?’. Revista Electrónica de Enseñanza de las Ciencias, Vol. $1 \mathrm{~N}^{\circ} 2$.

Matthews, M.R. (1991). Un lugar para la historia y la filosofía en la enseñanza de las ciencias. Comunicación, lenguaje y educación, 11-12, 141-155.

Meneses Villagrá, J.A. (1992). "Modelos didácticos con enfoque constructivista para la enseñanza de la Física en el nivel universitario". Revista Interuniversitaria de Formación del Profesorado.

Moreno, M. y Azcárate, C. (2003). "Concepciones y creencias de los profesores universitarios de matemáticas acerca de la enseñanza de las ecuaciones diferenciales". Enseñanza de las Ciencias.

Morrell, P.D. \& Lederman, N.G. (1998). "Students' attitudes toward school and classroom science: are they independent phenomena?" School Science \& Mathematics. 
Moscovici, S. (1985). "Psicología Social I, influencia y cambio de actitud. Cognición y desarrollo humano". Paidós, Barcelona.

Moscovici, S. y Hewstone, M. (1986). De la ciencia al sentido común. En Moscovici (comp.) 1986: Psicología Social. Tomo II. Barcelona: Paidós.

Papalia, D.C., Wendkos, S. y Duskin, R. (2001). Psicología del Desarrollo. $8^{a}$ Edición, Editorial McGraw-Hill. México.

Payne, M. (1985). "Using the Outdoors to Teach Science: A Resource Guide for Elementary and Middle School Teachers". New Mexico. State University. Educational Resources Information Center. Disponible en: http://www.eric.ed.gov/ERICDocs/data/ericdocs2sq1/content_storage_01/0000019b/80/2f/04/50.pdf

Porlán, R. (1993). "Constructivismo y escuela: hacia un modelo de aprendizaje basado en la investigación". DIADA, Sevilla.

Porlán, R. (2004) "Formulación de los contenidos escolares". Cuadernos de Pedagogía. 276, 65-70.

Pozo, I. y Gómez, M. (2006). "Aprender y enseñar ciencia: del conocimiento cotidiano al conocimiento científico”. Editorial Morata, España.

Pozo, I. y Gómez, M. (1998). Aprender y enseñar Ciencias. Madrid: Morata.

Rioseco, M. y Romero, R. (1999). "La contextualización de la enseñanza como elemento facilitador del aprendizaje significativo". Revista Paideia $\mathrm{N}^{\circ} 28$.

Rioseco, M. y Romero, R. (2000). "La contextualización de la Enseñanza de la Ciencia como elemento facilitador del aprendizaje significativo". Revista Paideia. Universidad de Concepción.

Rodríguez, A. (1991). "Psicología Social”. México: Trillas. ISBN.

Roger, L. (1998). "The Responsibility of Science", Universidad de Birmingham, Reino Unido. Fuente: International Journal of Science Education. Vol. 20, Nº 6 Fecha: Julio.

Silva Castro, E. (2003). "Enfoque de la Enseñanza de las Ciencias en el Nuevo Currículum de la Educación Nacional". Universidad Metropolitana de Ciencias de la Educación. Chile.

Trumbull, D., Scarano, G. \& Boney, R. (2006). "Relations among two teachers' practices and beliefs, conceptualizations of the nature of science, and their implementation of student independent inquiry projects". International Journal of Science Education.

Valerias, N. y Meneses Villagrá, J. (2005). "Modelo Constructivista para la Enseñanza de las Ciencias en línea". Enseñanza de las ciencias, Número Extra. VII Congreso. España.

Wu, Hsin-Kai \& Krajcik, J.S. (2006). "Inscriptional practices in two inquiry based classroom: a case study of seventh graders' use of data tables and graphs". Journal of Research in Science Teaching. 
